\title{
Atomistic Models and Molecular Dynamics
}

\author{
D. C. Sayle and T. X. T. Sayle \\ DEOS, Cranfield University, \\ Defense Academy of the United Kingdom \\ Shrivenham, Swindon, UK \\ Email: d.sayle@cranfield.ac.uk
}

\section{Atomistic Models and Molecular Dynamics}

\begin{abstract}
Here we show how atomistic computer simulation can help experiment unravel the rich structural complexity of oxide nanomaterials and, ultimately, aid the fabrication of nanomaterials with improved, tuneable or indeed new properties. We first explore the simulation methodologies: energy minimisation, monte-carlo, genetic algorithms and molecular dynamics together with the potential models used to describe the interactions between metal and oxide ions. These tools can be used to generate realistic structures that include all the essential microstructural features observed experimentally, such as surface structure (morphology, surface energy, faceting, surface steps, corners and edges), grain-boundaries and dislocations, intrinsic and extrinsic point defects and epitaxy. We show how the theoretician is able to capture all these (experimentally observed) structural details by attempting to simulate crystallisation. Equipped with realistic models, important properties can be calculated, including: electronic, chemical (catalytic activity, ionic diffusion and conductivity) and mechanical (hardness, elastic constants). This is illustrated by calculating the ease of oxygen extraction from the surface of a $\mathrm{CeO}_{2}$ nanocrystal compared with the bulk parent material with implications for oxidative catalysis. Throughout this chapter we emphasise the importance of molecular graphics - a much maligned and underrated tool - but without which, the generation of much of the simulation and experimental data would not have been possible.
\end{abstract}

Keywords: Interatomic potentials, evolutionary simulation, crystallisation, atom deposition, temperature assisted dynamics, nanocrystals, supported thin-films, encapsulated nanomaterials, nanopolycrystalline, dipolar surfaces, epitaxy, microstructure, properties. 


\section{Chapter 9 Atomistic Models and Molecular Dynamics}

9.1 Introduction

9.2 Theoretical methods

9.2.1 Interatomic potentials

9.2.2 Simulation strategies

9.2.2.1 Energy Minimisation

9.2.2.2 Monte Carlo

9.2.2.3 Genetic Algorithms

9.2.2.4 Evolutionary

9.2.2.5 Molecular Graphics

9.2.2.6 Molecular Dynamics

$9.3 \quad$ Nanoparticles

9.3.1 Amorphisation and Recrystallisation (A\&R) of $\mathrm{MnO}_{2}$

9.3.1.1 Microstructure

9.3.1.2 Evolution of the Atomistic Model

9.3.1.3 Final Structure

9.3.3 $\mathrm{CeO}_{2}$

9.3.3.1 Morphology

9.3.3.2 Evolution of the Atomistic Model

9.3.3.3 Dipolar surfaces

9.3.3.4 Oxidation of $\mathrm{CO}$ to $\mathrm{CO}_{2}$ using $\mathrm{CeO}_{2}$ nanoparticles

9.3.3.5 Reducibility of $\mathrm{CeO}_{2}$ nanoparticles using Monte Carlo

9.3.3.6 Aggregated nanoparticles and grain-boundaries

9.4 Supported Thin films

9.4.1 Ion deposition

9.4.1.1 $\mathrm{CaO} / \mathrm{MgO}(100)$

9.4.1.2 Growth Rates

9.4.1.3 Incommensuration

9.3.2 Near Coincidence Site Lattice Theory

9.3.2.1 $\mathrm{BaO} / \mathrm{MgO}$

9.3.2.2 $\mathrm{CeO}_{2} / \mathrm{Al}_{2} \mathrm{O}_{3}$

9.3.2.3 NiO/ZrO

9.4.3 Amorphisation and Recrystallisation

9.4.3.1 $\mathrm{SrO} / \mathrm{MgO}(100)$

9.4.3.2 $\mathrm{MgO} / \mathrm{BaO}(100)$

9.4.3.3 $\mathrm{CeO}_{2} / \mathrm{YSZ}$

9.4.4 Melting and Seeded-Crystallization

9.4.4.1 $\mathrm{FeO}$ (nanocrystalline)/ $\mathrm{FeO}$

9.4.5 Temperature Assisted Dynamics

9.4.5.1 BaO/SrO

9.5 Supported Nanoparticles

9.5.1 $\mathrm{MO} / \mathrm{BaO}(\mathrm{M}=\mathrm{Sr}, \mathrm{Ca}, \mathrm{Mg})$

9.6 Encapsulated Nanoparticles

9.7 Conclusions 


\subsection{Introduction}

A milestone for atomistic computer simulation will be the ability to model 'real' systems, which comprise, for example, $10^{23}$ atoms. This is unlikely to be realized in the near future, even with the almost exponential increase in computational power coupled with grid computing (for example see: [Schissel DP 2005]); at present 'only' trillions of atoms are possible. Accordingly, the simulator must resort to a variety of mechanisms such as imposing periodic boundary conditions or continuum descriptions of the material away from the site of interest rather than treat every ion explicitly. Perhaps the most important scientific field associated with the $21^{\text {st }}$ century is that of nanomaterials, which is rather agreeable to the atomistic simulator in that it is now possible to model every single ion comprising the system explicitly - at least in one dimension.

In this chapter we first describe briefly the theoretical methods, which include the interionic potentials used to describe the interactions between the oxygen and metal ions, together with the simulation strategies used to generate realistic atomistic structures. We have taken the liberty of ensuring the mathematics in this chapter is straightforward rather than rigorous. This is to help the non-expert gain a rudimentary understanding of atomistic simulation to help gauge its accuracy, scope and limitations without the pain of assimilating a forest of equations. We have, where necessary, provided references that are more rigorous for the interested reader.

Equipped with these theoretical methods, we show how they can be used to generate realistic atomistic models of oxide nanomaterials. We start with isolated nanoparticles of $\mathrm{MnO}_{2}$ and explore how microstructural features (such as dislocations, grain-boundaries, microtwinning, vacancies, substitutionals interstitials, intergrowths, morphology) can be introduced into the model. We next look at $\mathrm{CeO}_{2}$ nanoparticles and investigate how the morphology of the crystal changes as one traverses to the nanoscale. Here, the atomistic models provide insights into the possible structures of edges and corners, which comprise a high proportion of the surface area of the nanoparticle and are difficult to characterize experimentally. Edge and corner sites are also likely to be more reactive compared with ions accommodating plateau regions. At this point we take a short diversion to structural characterization and use the atomistic models to calculate important properties. In particular, the model is used to determine how easy it is to extract an oxygen ion from corner, edge or plateau sites of the nanoparticle compared to oxygen extraction from the surface of the parent (bulk) material.

Isolated nanoparticles have three dimensions at the nanoscale, whereas supported thin films have only one dimension - thickness - at the nanoscale. We show how simulation can be used to generate models for oxide supported oxide thin films. These models can then be used to predict the considerable influence that the substrate has in directing the structure of the thin film deposited thereon. Indeed, one can tune the properties of the thin film, by careful choice of substrate. We illustrate this by showing how the $\mathrm{CeO}_{2}(110)$ surface can be exposed in preference to the more stable $\mathrm{CeO}_{2}$ (111) by depositing a thin film of ceria on an yttrium stabilized zirconia substrate. This is important because the $\mathrm{CeO}_{2}(110)$ surface is catalytically more active compared with the $\mathrm{CeO}_{2}(111)$.

In the final section we investigate the structure of nanoparticles that are supported on a substrate and consider the structural implications of encapsulating a nanoparticle into a host oxide material.

\subsection{Theoretical Methods}

In this section we describe potential models that can be used to represent the interactions between the ions comprising the nanomaterial, the computational codes, used to perform the simulations, and 
finally, the strategies for generating atomistic models (atom coordinates) for the oxide nanomaterials.

\subsubsection{Interatomic potentials}

Many oxides are highly ionic and therefore we can describe them accurately using the Born model of the ionic solid [Stoneham AM 1985]. For nanomaterials, where a high proportion of the ions are located at a surface or interface, the interatomic potential must be reliable at describing ions in sites with low coordination. Clearly, the simplest way to address this is to use the same interatomic potential models to describe surface and interface ions as bulk ions. Indeed, this approach has enjoyed considerable success, for example see [Lewis GV 1985, Duffy DM 2004, Walker AM 2004]. Conversely, as (inevitably) systems become more complex and the need for higher levels of accuracy arise, surface specific potential models may play a more significant role in the future.

In the Born model of the ionic solid, all the ions are all assigned a charge. For a highly ionic material such as $\mathrm{MgO}$, formal charges (i.e. -2 for oxygen and +2 for magnesium) have been shown to work well. Conversely, for oxides that are less ionic, partial charges are sometime more appropriate. The (attractive) interaction energy between the charge on the oxygen and magnesium ion is given by:

$E_{\text {Atractive }}=\frac{q_{M g} q_{O}}{r_{M g-O}}$

Where $\mathrm{q}_{\mathrm{Mg}}$ and $\mathrm{q}_{\mathrm{O}}$ are the charges of the magnesium and oxygen ions respectively and $\mathrm{r}_{\mathrm{Mg}-\mathrm{O}}$ the distance between the two ions. Equation (9.1) suggests that the ions would prefer to move closer and closer to each other to achieve a more stable (lower energy) configuration. However, as the ions move closer to one another the 'electron clouds' of the two ions start to repel each other. This shortrange repulsion can be described using various equations, one of which is given below:

$\mathrm{E}_{\text {repulsive }}=\operatorname{Aexp}\left(-\mathrm{r}_{\mathrm{Mg}-\mathrm{O}} / \rho\right)$

There are two variable parameters associated with equation 9.2: A and $\rho$, which can be fitted to the experimental properties. For example, if one were to increase the value of parameter ' $A$ ', the repulsive interaction between the two ions increases and the ions would move further apart. Thus the A parameter used to describe $\mathrm{MgO}\left(\mathrm{a}_{\mathrm{MgO}}=4.2 \AA\right)$ may be smaller compared to the A parameter used to describe $\mathrm{BaO}$ which has a higher lattice parameter $\left(\mathrm{a}_{\mathrm{BaO}}=5.5\right)$.

Setting a particular value for A might give an accurate bond distance. However, ions are very rarely at their equilibrium distance and therefore one needs to describe the repulsive interaction between the two ions over a range of distances. Accordingly, the parameters A and $\rho$ are fitted together and to a range of experimental data. This may include, for example, the lattice parameter, elastic constants, dielectric constants etc. This enables the potential model to accurately describe the oxide over a range of interatomic distances and is particularly important when simulating the system at a particular temperature, other that $0 \mathrm{~K}$, where the ions are vibrating or indeed diffusing. Gale and Rohl provide a comprehensive treatment of atomistic potentials and how they can be derived [Gale JD 2003].

The total energy of the system is the sum of all the interactions between all pairs of ions comprising the oxide nanoparticle: 
$\mathrm{E}_{\text {total }}=\sum\left(\mathrm{E}_{\text {attractive }}+\mathrm{E}_{\text {repulsive }}\right)$

The energy is shown graphically for a diatomic molecule as a function of interionic separation in fig. 9.1. The minimum of the graph corresponds to the equilibrium bond distance where the attractive Coulombic term balances exactly the short range repulsion between the electron clouds. Starting from the equilibrium distance, as the ions move closer together, there is greater repulsion because of the electron clouds and the energy becomes more positive (less stable). Similarly, as the ions move further away from the equilibrium position, there is less repulsion from the electron clouds but also the Coulombic interaction is reduced and again the energy becomes more positive. The force, F, on the ions at each point can be calculated by measuring or calculating the gradient at a particular bond distance. At distances less than the equilibrium bond distance, the gradient (force) will be negative indicating that the force between the two ions tries to move them apart. Conversely, at distances greater than the equilibrium bond distance, the gradient will be positive and the force acts to move the ions closer together. At the minimum energy position, the force is zero.

In nature, the ions comprising the oxide nanoparticle will exist in a low or minimum energy configuration. This is not necessarily the lowest energy possible (global minimum energy [Gale JD 2003]) as most systems comprise, for example defects; rather the ion positions are such that the system exists in a 'local' energy minimum position. For a diatomic molecule it is easy to move the two ions into the lowest energy position (fig. 9.1). However, for a system comprising, for example, 10,000 or more atoms, the problem is more challenging. Indeed, there are many mechanisms for exploring low energy structures and five important methods are described briefly in the following section.

\subsubsection{Simulation Strategies}

\subsubsection{Energy Minimization [Gale JD 2003]}

Here, the ions are moved (iteratively) from a particular starting configuration to a lower energy configuration. This can be illustrated by considering the energy minimization of a diatomic molecule:

- $\quad$ Start with a (best guess) bond distance (fig. 9. 1)

- $\quad$ Calculate the gradient.

- If the gradient is negative reduce the bond distance, if it is positive, increase the bond distance.

- $\quad$ This process is repeated by recalculating the gradient until the gradient becomes zero, which corresponds to a minimum energy position.

In reality the problem is never quite this simple because a nanomaterial will have $3 N-6$ coordinates, where $N$ is number of atoms and therefore we have to minimise in $3 N-6$ - dimensional space. However, the basic procedure is that outlined above although there are many variations.

\subsubsection{Monte Carlo - MC [Mohn CE 2005, Harris DJ 2004, 2005]}

As the name implies, the ions are moved in a random fashion - determined by a roll of the dice! - to generate a low energy configuration. For example, a starting configuration is generated and then all the ions are moved in a random direction (determined by a roll of the dice). If the new configuration is lower in energy, the configuration is accepted. Conversely, if the configuration is higher in 
energy, the configuration is rejected. However, central to this method is that it allows the system to go uphill in energy as well as down and therefore escape from local energy minima that do not relate to realistic structures. In particular, instead of rejecting all structures that are higher in energy than the previous one, a further roll of the dice is performed. It is this second roll of the dice that decides whether a higher energy structure is indeed rejected.

\subsubsection{Genetic Algorithms - GA [Johnston RL 2003]}

Here, the atomistic structures of successive generations are constructed based upon attributes from the parent that are deemed desirable. Desirability in this sense means for example, low energy or realistic bond distances or chemically sound. Highly desirable configurations are assigned a high breeding probability and will likely generate lots of 'daughter structures' whereas undesirable structures are unlikely to 'procreate'. It is probably worth noting that daughter structures may have more than two parents!

\subsubsection{Evolutionary Simulation [Sayle DC 2003a]}

Here, the atomistic model is evolved in a similar fashion to experimental synthesis. For example, molecular beam epitaxy involves the deposition of molecules onto a substrate, which then adhere to its surface to form a coating or thin film. To generate the analogous model, instead of placing the whole of the thin film on top of the substrate in one go, the molecular deposition process is simulated. In this way the atomistic model evolves during the simulation and in so doing captures (hopefully) some of the structural features observed experimentally. Variations on this theme, and there are many, may include simulating the nucleation and growth of a material from solution or crystallization from a melt.

\subsubsection{Molecular Graphics}

This important, powerful, and sometimes overlooked, approach provides a wealth of visualization tools to move and manipulate atom coordinates in 3D. It is used as a GUI (Graphical User Interface) to construct chemically/physically or intuitively correct starting configurations prior to the simulation, to animate and thus 'observe' the simulation, to analyze final structures and also to help one comprehend complex (crystal) structures from experimental data (i.e. $x$ ray diffraction) that are difficult to understand in 2D.

\subsubsection{Molecular Dynamics - MD [Smith W 1996]}

This simulation strategy enables one to simulate the movement of the ions comprising the system at a particular temperature and as a function of time. The method uses Newtons laws of motion to calculate and change the positions of all the ions as they move, vibrate or collide with one another. For example, the force, $\mathrm{F}$, acting upon a particular ion, $\mathrm{i}$, at time, $\mathrm{t}$, is given by:

$F_{i}(t)=m_{i} a_{i}(t)$

Where, $\mathrm{m}_{\mathrm{i}}$ is the mass of the ion and $\mathrm{a}_{\mathrm{i}}$, its acceleration. At the start of the simulation, the positions $\mathrm{r}_{\mathrm{i}}(\mathrm{x}, \mathrm{y}, \mathrm{z})$ of all the ions, comprising the nanoparticle, are defined. The forces acting upon each ion can then be calculated by adding up all the pair-wise interactions between all the ions comprising the oxide nanoparticle using an interatomic potential such as that described in equation 9.3. The force, which is the gradient of the energy graph, fig. 9.1, is given by:

$$
F=\frac{d E}{d r}
$$


First, the accelerations of the ions can be calculated by rearranging (9.4) and dividing by the mass of the ion:

$\mathrm{a}_{\mathrm{i}}(\mathrm{t})=\mathrm{F}_{\mathrm{i}}(\mathrm{t}) / \mathrm{m}_{\mathrm{i}}$

Once the accelerations have been calculated, the velocities can be updated after a particular time interval, $\delta \mathrm{t}$ :

$\mathrm{v}_{\mathrm{i}}(\mathrm{t}+\delta \mathrm{t})=\mathrm{v}(\mathrm{t})+\mathrm{a}_{\mathrm{i}}(\mathrm{t})(\delta \mathrm{t})$

Finally, the coordinates of the atom positions can be changed based upon the atom velocities:

$r_{i}(t+\delta t)=r_{i}(t)+v_{i}(t)(\delta t)$

At this point all the required information has been calculated and the cycle repeats - i.e. the force, equation 9.5, is recalculated with the new atomic coordinates.

The time interval, $\delta \mathrm{t}$, used for a particular simulation, has to be chosen carefully and must be smaller than the time required for a bond to vibrate. This is to ensure that the energy and forces are updated before the ions have a chance to move too near to one another. Unfortunately, a bond vibration is of the order of $10^{-15}$ seconds and therefore the biggest limitation of MD simulation is the short period of time one can run the simulation. For example, to simulate just one second in real time would require the calculation of over $10^{15}$ cycles, which is, at present, well beyond even the fastest computer processors. Moreover, parallelization of the problem does not really help as the equations have to be solved sequentially and therefore introducing more parallel processors only enables the number of ions in the system to be increased, rather than facilitating any increase in the simulation time. However, as one might have anticipated, various innovative and imaginative methods have been introduced to help counter this somewhat debilitating limitation.

Many computational codes have been written to allow the user to perform MD simulations (for example: collaborative computational projects, www.ccp.ac.uk). For most of the work described in this chapter the DL_POLY code was used to perform the dynamical simulations [Smith W 2002]. This (parallel) code was developed by Daresbury Laboratory in the mid 1990s for the molecular simulation community in the United Kingdom. After more than 20 years since its inception, this code enjoys a world-wide user base. Indeed, over 600 licenses have been issued. A philosophy, central to the DL_POLY MD code, is that its source code is freely available [Smith W 1996] and therefore the users can modify, add and share new subroutines to the code to suite their particular simulation. As such, the code has been continually evolving since its inception.

In practice, it is rare that any one simulation technique is used in isolation; rather the methods are combined (especially with molecular graphics), which results in a more powerful technique.

In this section we have provided a brief and necessarily simple overview of some atomistic simulation methods and procedures. For a more in depth and authoritative treatment, we suggest that the interested reader refer to 'Molecular Modeling, Principles and applications' by Andrew R Leach [Leach AR 2001], which provides an excellent and detailed reference source. In the following section we describe how the simulation techniques are applied to the study of oxide nanomaterials. 


\subsection{Nanoparticles}

In this section, we describe the evolutionary simulation procedure, used to generate the atomistic models described in this chapter, in more detail and illustrate the procedure using $\mathrm{MnO}_{2}$ as an example. Next, we explore the structure, morphology and catalytic activity of $\mathrm{CeO}_{2}$ nanoparticles and compare with the parent material.

\subsubsection{Amorphisation and Recrystallisation (A\&R) of $\mathrm{MnO}_{2}$}

Many atomistic studies, used to investigate the structure and energetics of a system, proceed by defining the basic atomistic structure ('by-hand'), which is then simulated using static or dynamical methods. However, for systems that comprise complex 3D microstructures, such as nanoparticles, the starting structural models can prove challenging to generate. For example, the simulator needs to include within the atomistic model the morphological structure, including the particular low energy surfaces that are exposed. And whilst there are many simulation codes available to generate individual surfaces (such as ref: [Gay 1995]), for a nanoparticle one also needs to consider the atomistic structure of edges, where a pair of surfaces meet and vertices, where three or more surfaces meet, together with the implications of dipolar surfaces [Harding JH 1999]. One also requires a representation of the defect chemistry, which may include point defects that exist within the bulk or surface regions of the nanoparticle including, both intrinsic and extrinsic defects such as vacancies, interstitials and substitutionals together with clustering or segregation of these defects. For larger nanoparticles, defects such as dislocations and/or grain boundaries are likely to be present. And whilst the introduction and simulation of a specific and isolated grain-boundary or dislocation is now routine within the perfect 'bulk' material [Duffy DM 1986, Walker AM 2004], it remains challenging to introduce these into, for example, a 50,000-atom nanoparticle.

All these structural and microstructural features are likely to influence, or indeed govern, the properties of the oxide nanomaterial. Accordingly, if the simulator is to offer predictions of a nanoparticle that are both accurate and can be used reliably by the experimentalist, the atomistic models must include all the microstructural features alluded to above. To introduce all these features 'by-hand' is certainly a daunting if not intractable a prospect and therefore an alternative simulation strategy must be sought to generate models with this complexity.

Materials synthesis inevitably involves some kind of 'crystallisation' process. Indeed, it is the crystallisation process that controls the (micro)structure and hence the properties of the material. Moreover, by modifying the crystallisation process (whether crystallisation from solution, vapour deposition, molecular beam epitaxy, ball milling etc.) one can exact some control over the microstructure and hence the properties of the material. Clearly, the ideal way of capturing, within a single atomistic model, all the microstructural features observed experimentally, is to simulate the crystallisation process itself. Indeed, there are many theoretical studies that explore the crystallization process. For example Piana and Gale performed some highly detailed MD simulations on the growth and dissolution of urea crystals [Piana S 2005]. Similarly, Hamad et al. used MD to explore the embryonic stages of ZnS nanobubbles [Hamad S 2005]. These outstanding and elegant dynamical atomistic simulations capture much of the important features associated with the nucleation, growth and dissolution processes. Indeed, these approaches would prove ideal in generating models for nanoparticles. Unfortunately, they would also prove far too computationally expensive (at present) to use them to generate models for oxide nanoparticles comprising, for example, 50,000 atoms. An alternative approach to this problem, and one that can routinely accommodate $100,000+$ atoms with the computational facilities available today, is Amorphisation and Recrystallisation (A\&R) [Sayle DC 1999 and Sayle TXT 2005b]. We illustrate this evolutionary technique in the following section by considering the example of $\mathrm{MnO}_{2}$ nanoparticles. 


\subsubsection{Microstructure}

$\mathrm{MnO}_{2}$ is a widely studied electrochemical material that accommodates over 14 polymorphs [Chabre $Y$ 1995] and comprises an internal interconnecting tunnel structure with channel sizes commensurate with that of cations spanning $\mathrm{H}^{+}$to $\mathrm{Cs}^{+}$. Accordingly, they can act as host lattices for the insertion, storage and extraction of cations and hence charge, which is central to their ability to store energy. Among the several varieties of $\mathrm{MnO}_{2}$, the form known as $\gamma-\mathrm{MnO}_{2}$ exhibits the best electrochemical activity. This material comprises a wealth of microstructural features. Indeed, it has been proposed that this rich microstructure facilitates the exemplary properties of this material. The microstructural features includes: de Wolff disorder, which is the intergrowth of domains comprising single and double channels, microtwinning and a variety of point defects such as cation vacancies. Clearly, the ability of $A \& R$ to evolve, from an amorphous precursor, the crystalline $\mathrm{MnO}_{2}$ structure together with microstructural features observed experimentally is a stringent validation of the $A \& R$ strategy and provides a useful demonstration of the technique. In the following section we describe briefly the generation and structure of $\mathrm{MnO}_{2}$ nanoparticles about $10 \mathrm{~nm}$ in diameter. Further details have been published elsewhere [Sayle TXT 2005b].

\subsubsection{Evolution of the Atomistic Model}

A 'cube' of $\mathrm{MnO}_{2}$, comprising about 25,000 atoms, was constructed and is shown in fig. 9.2(a). The coordinates of all the ions comprising this cube were then changed to increase the lattice parameter by $36 \%$ and $\mathrm{MD}$ was then performed on this system at $2000 \mathrm{~K}$. The $\mathrm{MnO}_{2}$ can perhaps be compared with an elastic band, which is tensioned and then released. As soon as MD is applied to this tensioned configuration, the $\mathrm{Mn}$ and $\mathrm{O}$ ions 'implode'. And as they accelerate and move towards the centre of the cube the hit other ions and bounce off. The culmination of all the accelerations and collisions results in the amorphisation of the $\mathrm{MnO}_{2}$, which is shown in fig. 9.2(b). Essentially, the energy introduced into the system to tension the nanoparticle, results in its amorphisation. During prolonged MD simulation, the $\mathrm{MnO}_{2}$ nanoparticle starts to recrystallise. Snapshots of the recrystallisation are shown in fig. 9.3(a-f) together with the energy of the system in fig. 9.3(g): From 0-1000ps the $\mathrm{MnO}_{2}$ is amorphous. At about 1000ps, a crystalline seed evolves within the amorphous $\mathrm{MnO}_{2}$ and the $\mathrm{Mn}$ and $\mathrm{O}$ ions start to condense onto the surface of this seed increasing its size, fig. 9.3(a, b). The crystallization front propagates from this seed to encompass the whole nanoparticle; recrystallisation is essentially complete after about 3000ps. The energy of the system goes down during the recrystallisation with the difference in energy between the amorphous structure and crystalline structure reflecting the latent heat of crystallisation. Finally, the nanoparticle is cooled, under MD, to $0 \mathrm{~K}$. The complete simulation is computationally intensive and required about 5 days using 96 processors of a SunFire F15K Galaxy-class configuration supercomputer.

\subsubsection{Final Structure}

Inspection of the final, 0K structure, shown in fig. 9.4, reveals a crystalline nanoparticle, which accommodates predominantly domains that confirm to the pyrolusite structure [Chabre Y 1995] - a low-energy polymorph of $\mathrm{MnO}_{2}$. Fig. 9.4(a) shows a representation of the $\mathrm{Mn}$ and $\mathrm{O}$ atom positions comprising the nanoparticle. The oxygen sublattice forms a close packed array and gives rise to octahedral sites, $50 \%$ of which are filled by $\mathrm{Mn}$ to give the $\mathrm{MnO}_{2}$ stoichiometry. In particular, it is the filling of these octahedral sites that gives rise to the particular polymorph. In fig. 9.4(a) a rectangle has been drawn that encompasses two oxygen planes and an atomic plane of $\mathrm{Mn}$ sandwiched between. These three planes are shown (plan view) in fig. 9.4(b). Inspection of the filling by $\mathrm{Mn}$ reveals straight single parallel lines of $\mathrm{Mn}$, which correspond to the pyrolusite polymorph. These lines also change direction, which corresponds to microtwinned domains as observed experimentally [Hill MR 2004]; an enlarged segment is depicted in fig.9.4(c). Detailed 
analysis of the final structure, using graphical techniques, reveals also the presence of vacancies, which have also been observed experimentally.

In summary, $A \& \mathrm{R}$ has been used to generate models for $\mathrm{MnO}_{2}$ nanoparticles that are realistic in that they reflect the low-energy polymorphic configuration (pyrolusite) and include complex microstructural features that have been observed experimentally. Accordingly, we suggest therefore that the A\&R strategy is an appropriate simulation tool one can use to generate realistic models of oxide nanoparticles.

\subsection{2 $\mathrm{CeO}_{2}$}

The second isolated nanoparticle that we consider is ceria, $\mathrm{CeO}_{2}$. This material has enjoyed much attention recently because its high oxygen conductivity, at moderate temperatures, makes it a potential candidate as a component of a fuel cell [Yu HB 2005]. Here, we explore its catalytic properties.

In the early 1980s, three way catalysts (TWC) were found to have the ability to oxidise CO and hydrocarbons, while simultaneously reducing $\mathrm{NO}_{\mathrm{x}}$ to form less toxic products such as $\mathrm{CO}_{2}, \mathrm{H}_{2} \mathrm{O}$ and $\mathrm{N}_{2}$. These conversions are attained in a narrow window of the air-to fuel ratio [ Di Monte $\mathrm{R}$ 2005]. $\mathrm{CeO}_{2}$, is a common promoting component in TWC, which has been attributed to its low $\mathrm{Ce}^{4+} / \mathrm{Ce}^{3+}$ redox potential and high oxygen defect mobility, but what are the implications for this material when one traverses down to the nanoscale?

\subsubsection{Morphology}

The atomistic structure of $\mathrm{CeO}_{2}$ nanoparticles has been well characterized by several groups using transmission electron microscopy [Wang ZL 2003, Zhang F 2004]. In particular, the shape of $\mathrm{CeO}_{2}$ nanoparticles, about $10 \mathrm{~nm}$ in diameter, can be described as conforming to truncated octahedra. Specifically, the nanoparticles were observed to expose $\{111\}$ surfaces, truncated by $\{100\}$ - within a TEM micrograph, these polyhedra project into hexagons. TEM images of these nanoparticles are presented (with permission) in fig. 9.5(b) together with a schematic illustrating the proposed morphological shape, based upon the TEM micrographs.

Atomistic simulation can be used to predict the crystal morphology by calculating the energy of low index surfaces and this method has been shown to be remarkably accurate. Intuitively, the more stable the surface, the more that particular surface is exposed in the morphology. Indeed, this strategy has been shown to be a powerful simulation tool and has enjoyed much success [TITILOYE JO 1991] not least because of its inherent simplicity. The calculated energies of the low index surfaces of $\mathrm{CeO}_{2}$ are $(111)>(110)>(100)$ and therefore the simulation would, in accord with experiment, predict $\{111\}$ to dominate [Vyas S 1998]. Surface energy calculations also predict that the morphology of $\mathrm{CeO}_{2}$ should comprise more $\{110\}$ than $\{100\}$. However, this is not supported experimentally; rather the only other face observed is the $\{100\}$, which is surprising because this surface is dipolar [Harding JH 1999].

It is inevitable that a simplistic simulation approach, such as predicting crystal morphologies based solely on surface energy calculations, will fail in certain circumstances. This is because crystal morphologies can be preparation dependent [Chen HI 2005]. Accordingly, simulation strategies, used to predict crystal morphologies, must include features pertaining to nucleation and growth [Rohl AL 2004]. This added complexity may facilitate more accurate predictions but at a cost of loosing simplicity of approach. 
In the following section we describe how $A \& R$ can be used to predict the crystal morphology of $\mathrm{CeO}_{2}$ nanoparticles. This should also prove a useful validation of the A\&R method because detailed TEM images are available to compare. It is worth bearing in mind that the A\&R method has to accommodate two unexpected features associated with the morphology of $\mathrm{CeO}_{2}$. First the nonexistence of (relatively low-energy) $\mathrm{CeO}_{2}\{110\}$ surfaces, and second the ability for A\&R to evolve $\{100\}$ surfaces and necessarily quench the dipole, which is associated with the (100) surface.

\subsubsection{Evolution of the Atomistic Model}

$\mathrm{A} \& \mathrm{R}$ was used to predict the morphological shape and atomistic configuration of $\mathrm{CeO}_{2}$ nanoparticles about $8 \mathrm{~nm}$ in diameter. Full details regarding the simulation can be found elsewhere [Sayle TXT 2004] and follow a very similar procedure as that described above for $\mathrm{MnO}_{2}$ nanoparticles. The morphologies of the $\mathrm{CeO}_{2}$ nanoparticles, predicted using $\mathrm{A} \& \mathrm{R}$, are shown in fig. 9.5(a); TEM images are also shown, fig. 9.5(b), as a comparison. The theoretical models do indeed exhibit truncated octahedral morphologies in accord with experiment and, in contrast to surface energy predictions, the predicted morphologies do not (at first sight) expose $\{110\}$ surfaces. However, upon closer inspection of the atomistic models, fig. 9.5(a), we note that the edges, formed where two $\{111\}$ surfaces intersect, could perhaps be described as $\{110\}$. Also, steps on the $\{111\}$ also appear commensurate with $\{110\}$. TEM images are primarily $2 \mathrm{D}$ projections and therefore structural features that are '3D' are difficult to identify experimentally. Accordingly, we suggest that the atomistic models, which can be observed and manipulated in 3D using graphical techniques, are valuable in that they can be used to help interpret TEM images and therefore aid experiment in characterizing the full 3D atomistic structure of $\mathrm{CeO}_{2}$ nanoparticles.

\subsubsection{Dipolar Surfaces}

Fluorite-structured $\{100\}$ surfaces are dipolar and therefore inherently unstable [Norenberg $\mathrm{H}$ 2001]. Surface energy calculations on dipolar surfaces have necessitated quenching the dipole prior to simulating the system with static or dynamical methods, which is normally achieved by physically rearranging the ions 'by-hand' [Stanek CR, 2004]. Conversely, A\&R is a simulation method in which the structure/atom positions evolve during the simulation. Accordingly, this approach does not need (or indeed allow!) the simulator to manually move ions with an aim of quenching the dipole. However, close inspection of the $\mathrm{CeO}_{2}\{100\}$ at the end of the simulation, fig. 9.6, revealed a 50\% reduction in the number of oxygen ions at the $\{100\}$ surfaces. Specifically, the simulation has evolved a structure in which the dipoles associated with the $\{100\}$ surfaces have been quenched. Moreover, because these surfaces have evolved, we suggest that they are likely to be more realistic than dipolar surfaces that have had their dipoles quenched manually (based upon chemical intuition) because the simulated crystallization (A\&R) reflects (in part) crystallization that occurred during the experimental synthesis. However, this assumption must be tested on a system where the real structure of the (quenched) dipolar surface is unambiguous - down to the individual positions of the surface ions, which is, at this time, a contentious issue [Norenberg H 2001].

\subsubsection{Oxidation of $\mathrm{CO}$ to $\mathrm{CO}_{2}$ using $\mathrm{CeO}_{2}$ nanoparticles}

Once a realistic model of the $\mathrm{CeO}_{2}$ nanoparticle has been generated, this model can be used to predict its catalytic activity. In particular, atomistic simulation can be used to predict whether the nanoparticle would be catalytically more or less active compared with the parent material. For example, the oxidation of $\mathrm{CO}$ to $\mathrm{CO}_{2}$ involves extracting oxygen from the surface of $\mathrm{CeO}_{2}$. $\mathrm{Clearly}$, if one could make the surface oxygens more facile to extraction, one can, potentially, fabricate a more active catalyst. It has been shown previously, that extracting oxygen from the step site of $\mathrm{CeO}_{2}$ is easier than from the plateau [Sayle TXT 1992, 1994]. This appears intuitive in that an oxygen ion accommodating a step site is lower coordinated compared with an oxygen ion at a plateau site and therefore less strongly bound to the surface. One can then argue that if more step 
sites can be generated at the surface of the catalyst then, theoretically, one should be able to generate a more active catalyst. One way of achieving this (discussed later) is to deposit a thin film of $\mathrm{CeO}_{2}$ on a substrate, which acts as a template in directing the $\mathrm{CeO}_{2}$ to expose a reactive surface. The other approach is to traverse to the nanoscale:

At risk of stating the obvious, the dictionary definition of a polyhedron is: a solid figure consisting of more than four plane faces. A pair of faces will meet along an edge and three or more edges meet at a vertex. Accordingly, as a particle decreases in size, the proportion of ions accommodating edge and vertex sites, compared to those occupying plateau regions, increases. In addition, the number of surface to bulk atoms also increases. Eventually - at the nanoscale - the number of edge sites becomes commensurate with plateau sites and surface ions commensurate with bulk ions. Indeed, one can perform a simple count of the ions in the $\mathrm{CeO}_{2}$ nanoparticle, fig. 9.5(a), to confirm this. Accordingly, the $\mathrm{CeO}_{2}$ nanoparticle should comprise a high concentration of labile (lowcoordinated) surface oxygen species.

To explore the catalysis of the $\mathrm{CeO}_{2}$ nanoparticle, we need to determine how difficult it is to extract an oxygen ion from its surface. The simulation procedure that can be used to calculate this is relatively straight-forward. In particular, we choose an oxygen ion to remove, we then extract this ion out of the surface and replace two $\mathrm{Ce}^{4+}$ with $\mathrm{Ce}^{3+}$ (to accommodate for the charge imbalance). The energy of this system is then calculated and compared with the energy of the same system but with an oxygen ion removed from a different position. Using this procedure one can generate a table of oxygen location vs. vacancy formation energy. However, when one inspects the atomistic model, fig. 9.5, a daunting number of different surface oxygen species are available for extraction; the nanoparticle comprises 15,972 ions, many of which are surface or near surface species. Accordingly, an oxygen vacancy formation energy 'averaged' over the whole nanoparticle was calculated. Specifically, 266 oxygens were removed from the surface of the starting (prior to amorphisation) configuration together with $532 \mathrm{Ce}^{4+}$ species replaced by $\mathrm{Ce}^{3+}$. The rationale underlying introducing the defects prior to the amorphisation is that the A\&R will direct the vacancies and $\mathrm{Ce}^{3+}$ species into a range of low-energy configurations thereby providing a more realistic average reduction energy for the nanoparticle. The nanoparticle comprises, in total, 15,972 ions and therefore removal of 266 oxygens results in a reduced nanoparticle with composition $\mathrm{CeO}_{1.95}$. This system was amorphised and then recrystallised; further details pertaining to the simulation can be found elsewhere [Sayle TXT 2005a]. The final structure is shown in fig. 9.7.

Inspection of the reduced nanoparticle, fig. 9.7(a-c), reveals an octahedral morphology comprising $\{111\}$, truncated by $\{100\}$, which is similar to the unreduced nanoparticle, fig. 9.5. The $\mathrm{Ce}^{3+}$ ions can be seen to decorate step and corner sites in addition to positions on $\{111\}$ terraces. A simple count revealed that $20 \%$ of the total number of $\mathrm{Ce}^{3+}$ species occupy positions on $\{111\}$ terraces, $26 \%$ decorate $\{111\}$ step $(17 \%)$ or corner $(9 \%)$ positions and $11 \%$ occupy positions on $\{100\}$. The remaining $43 \%$ of the $\mathrm{Ce}^{3+}$ species occupy positions within the bulk of the nanoparticle. We note, however, that most of these 'bulk' $\mathrm{Ce}^{3+}$ species occupy positions one or two atomic planes below the surface rather than deep inside and near the centre of the nanoparticle. Locating oxygen vacancies is much more difficult compared with locating $\mathrm{Ce}^{3+}$ species because a vacancy is not a physical entity within an atomistic model, and the relaxation of the lattice surrounding an $\mathrm{O}^{2-}$ vacancy or $\mathrm{Ce}^{3+}$ is sometimes considerable. Consequently, a simple count of the vacancies, together with their location, was not possible, although oxygen vacancies were identified to occupy step and corner sites in addition to $\{111\}$ terrace sites. In addition, oxygen vacancies were observed to lie one oxygen subplane below the surface oxygen ions. 
The energy required to extract an oxygen ion from the bulk and from the 'perfect' (111), (110) and (310) surfaces of $\mathrm{CeO}_{2}$ was calculated by Sayle, Parker and Catlow [Sayle TXT 1992, 1994]. From these values, the energy associated with oxidizing carbon monoxide to carbon dioxide, using an oxygen ion extracted from a ceria surface, was calculated following:

$$
\mathrm{CO}(g)+\mathrm{CeO}_{2} \rightarrow \mathrm{CO}_{2}(g)+\mathrm{V}_{\mathrm{O}}^{\bullet \bullet}+2 \mathrm{Ce}_{\mathrm{Ce}}^{\prime}
$$

These values are presented, together with the average energy required to extract an oxygen ion from the surface of a $\mathrm{CeO}_{2}$ nanoparticle to oxidize the carbon monoxide, in table 9.1. We note that the more negative the energy the more labile the oxygen and therefore the easier it is to remove from the lattice to promote the oxidation of $\mathrm{CO}$.

The simulations suggest that it is easier to extract oxygen from the surface of a $\mathrm{CeO}_{2}$ nanoparticle compared with the bulk parent material. In particular, the average energy required to extract an oxygen ion from the surface of the ceria nanoparticle is lower compared with the (most stable) $\mathrm{CeO}_{2}$ (111) surface associated with the parent material. Moreover, the average energy required to extract oxygen from the nanoparticle is calculated to lie between those energies calculated previously for extracting an oxygen from the bulk, (111), (110) or (310) surfaces. This we suggest is a realistic value because fig. 9.7 reveals that $\mathrm{O}^{2-}$ vacancies and $\mathrm{Ce}^{3+}$ ions populate all conceivable positions in the nanoparticle including 'bulk', terrace and step sites and comprise a variety of configurations spanning simple isolated defects to complex multi-defect clusters. For illustration, the atom positions showing the structure of three defect clusters are shown in fig. 9.7(d-f)

In conclusion, the simulations predict that ceria nanoparticles can offer more reactive surfaces compared to the parent material, because of the higher proportion of step/corner sites, and therefore facile oxygen species. Accordingly, ceria nanoparticles may help promote the oxidation of $\mathrm{CO}$ to $\mathrm{CO}_{2}$, which is central to the performance of ceria based three-way catalysts.

\subsubsection{Reducibility of $\mathrm{CeO}_{2}$ nanoparticles using Monte Carlo}

In 1996, Cordatos, Ford and Gorte generated atomistic models for $\mathrm{CeO}_{2}$ nanoparticles $\mathrm{Ce}_{\mathrm{n}} \mathrm{O}_{2 \mathrm{n}}(\mathrm{n}=2$ 20 and $n=50$ ) using a Monte Carlo approach coupled with simulated annealing [Cordatos H 1996]. The authors found that the $\mathrm{Ce}_{50} \mathrm{O}_{100}$ nanoparticle exhibited the fluorite structure and exposed the (111) surface. The reducibility of the clusters was determined from the difference in lattice energies of $\mathrm{Ce}_{\mathrm{n}} \mathrm{O}_{2 \mathrm{n}}$ and $\mathrm{Ce}_{\mathrm{n}} \mathrm{O}_{2 \mathrm{n}-1}$, where charge neutrality was maintained by changing two $\mathrm{Ce}^{4+}$ ions to $\mathrm{Ce}^{3+}$. The authors concluded that the reduction of ceria nanoparticles is structurally sensitive and that larger crystals are more difficult to reduce.

\subsubsection{Aggregated nanoparticles and Grain-boundaries}

Experimentally it is well-known that during the synthesis of oxide nanoparticles the nanoparticles are not always monodispersed; rather they aggregate together - as shown by HRTEM for example see Ref [Wang ZL 2003]. The interfacial structure between the two nanoparticles can (for example) be coherent (if perfectly aligned), can form a twin-boundary (if they are aligned but miss-oriented by a particular angle) or form a more general and complex grain boundary structure. Examples (HRTEM) of all such grain-boundary structures can be found in [Wang ZL 2003, Zhang F 2004]. The properties of a grain-boundary have been shown to differ profoundly compared with the perfect parent material [Sata N 2001, Duffy DM 1986, Sayle DC 2005]. Clearly, for aggregated nanoparticles, the number of grain-boundaries will be considerable. Accordingly, if one is to make predictions pertaining to the properties of nanoparticles then models for nanoparticles that comprise grain-boundaries must be generated. And using atomistic computer simulation it is possible to generate such structures. For example, fig. 9.8 shows a model for a $\mathrm{CeO}_{2}$ nanoparticle that 
comprises two twin-boundaries. This nanoparticle, which comprises about 21,000 atoms, was generated using an analogous procedure as that used to generate $\mathrm{MnO}_{2}$ or $\mathrm{CeO}_{2}$ nanoparticles, described above.

\subsection{Supported Thin Films}

Thus far we have explored, using atomistic simulation, the structure of 'isolated' nanoparticles and find the structure and chemistry to be quite different compared with their bulk counterparts. In the next section we consider oxide thin films supported on an oxide substrate. Here, in contrast to a nanoparticle, which has all three dimensions at the nanoscale, the thin film exists at the nanoscale in only one dimension - its thickness.

\subsubsection{Ion Deposition}

Atomistic models for oxide thin films with nanoscale thicknesses, supported on an oxide substrate, can be generated by depositing those ions comprising the oxide thin film, onto the surface of the substrate using MD. This type of approach can be classed as evolutionary and reflects or simulates the basic operation of experimental deposition techniques such as molecular beam epitaxy. We illustrate the technique using $\mathrm{CaO} / \mathrm{MgO}(001)$ as a model system.

\subsubsection{1 $\mathrm{CaO} / \mathrm{MgO}(100)$}

Snapshots (taken during MD simulation) depicting the growth of $\mathrm{CaO}$ deposited on $\mathrm{MgO}(100)$ are shown in fig. 9.9. More details pertaining to this study can be found in reference [Sayle DC 1999a]. Initially, ions are fired from a source to the surface of the $\mathrm{MgO}$ substrate and as the ions hit the surface, they move, under MD, into low energy configurations. For example, cations and anions diffuse across the surface via a hopping mechanism and if they come in close contact, they are likely to adhere to form a diatomic. Further ions 'diffusing' across the surface will add progressively to this diatomic to form a larger cluster. The structure of this $\mathrm{CaO}$ cluster is clearly influenced profoundly by the interactions from the underlying substrate. Eventually, with continued deposition, a second layer will start to evolve followed by a third and so forth. It is interesting to note that the $\mathrm{Ca}$ and $\mathrm{O}$ ions swap positions with $\mathrm{Mg}$ and $\mathrm{O}$ ions of the underlying $\mathrm{MgO}(100)$ substrate. This is because an energetically facile surface diffusion mechanism involves an exchange process between the deposited ions and ions occupying the surface atomic layer of the substrate. This suggests that ionic materials may not be grown on a substrate with a similar structure without significant intermixing across the interfacial regions. This phenomenon was explored in more depth by Harris and co-workers [Harris DJ 2005].

The nature of the substrate, on which the ions are deposited, also has a significant influence on the structure of the oxide thin film deposited thereon. For example, substrates that comprise a high concentration of surface steps, provide energetically favourable nucleation sites for ions deposited thereon. This is because of the increased coordination of an ion that adheres at a step, compared with a plateau position. The surface(s) exposed by the substrate will also influence profoundly the thin film because the substrate can act as a template in directing the structure of the overlying thin film [Sayle DC 2002a]. This is especially relevant for films with nanothicknesses, because the interfacial interactions will comprise a significant component of the total energy.

\subsubsection{Growth Rates}

A major limitation with MD, in the context of ion deposition, is the timescale generally accessible, which, using (present day) computational resources is still only of the order of nanoseconds. If the whole of the thin film is deposited within this timescale, this corresponds to thin film growth rates of at least meters per second; experimentally, depositions are typically of the order of 'atomic layers 
per second'. One solution to reduce the simulated deposition speed is to increase the temperature of the simulation. This increases the diffusivity of the ions in the simulation to levels commensurate with experiment (at a much lower and more realistic a temperature). However, one must be cautious in using this approach because at high temperatures the diffusion mechanisms may change, which may result in artificial structures. Methods for circumventing timescale issues, pertaining to MD simulation, are receiving considerable attention at present, and various innovative strategies are being devised - see, for example, [Voter AF 2002, Harris DJ 2004].

\subsubsection{Incommensuration}

Another important issue associated with simulating oxide thin films with nanoscale thicknesses is the incommensurate relationship that exists between the lattice parameter of the substrate and thin film deposited thereon. For example, consider the $\mathrm{CaO} / \mathrm{MgO}$ system in which the lattice parameters of the component materials are: $\mathrm{a}_{\mathrm{CaO}}=4.8 \AA ; \mathrm{a}_{\mathrm{MgO}}=4.2 \AA$. To create a model for a $\mathrm{CaO}$ thin film supported directly on top of the $\mathrm{MgO}$ substrate with all ions in alignment (coherent interface - see Sutton AP 1995]), would require the $\mathrm{CaO}$ to be compressed by about $13 \%$. This is easily achievable for a single atomic layer because the energetically favourable cation-anion interactions across the interface will compensate for the energy required to strain a single atomic layer of $\mathrm{CaO}$ to bring it into alignment with the underlying $\mathrm{MgO}$ substrate. However, for each additional layer of $\mathrm{CaO}$ added, an extra strain energy term would also need to be added. Eventually, at a particular critical thickness [Dong L 1998, Lu YF 2005], the strain energy would outweigh the energy associated with the interactions across the interface between the $\mathrm{CaO}$ and $\mathrm{MgO}$ [Schnitker J 1998]. Above the critical thickness, the structure and configuration of the overlying thin film may change with respect to the underlying substrate to reduce the strain. (Micro)structural changes include, for example, the formation of superlattices, misfit dislocations, low interfacial densities, grain-boundaries etc [Chambers SA 2000]. Clearly, atomistic simulation must be able to reproduce these microstructural features if reliable predictions are to be made using these models. And in the following section we describe how such microstructural features can be introduced into the atomistic models.

\subsubsection{Near Coincidence Site Lattice (NCSL) theory}

One way of addressing the difference (incommensurate relationship) between the lattice parameter of the thin film and that of the substrate, is to use a near coincidence site lattice theory to predict epitaxial relationships with low associated misfits. In the following sections we describe how this approach is used, in conjunction with atomistic simulation, to generate models of thin films.

\subsubsection{BaO/MgO}

Sayle and co-workers [Sayle TXT 1993] used a NCSL theory, coupled to atomistic simulation, to predict superlattices for $\mathrm{BaO}\left(\mathrm{a}_{\mathrm{BaO}}=5.5 \AA\right)$ thin films supported on $\mathrm{MgO}\left(\mathrm{a}_{\mathrm{MgO}}=4.2 \AA\right)$. For example, for the $\mathrm{BaO}(100) / \mathrm{MgO}(100)$ system, $10 \mathrm{BaO}$ unit cells $(55.0 \AA)$ of the thin film are nearly lattice matched with $13 \mathrm{MgO}$ unit cells $(54.6 \AA)$ of the substrate. Clearly, 54.6 and $55.0 \AA$ are in near coincidence and the $\mathrm{BaO}$ overlayer only needs to be expanded by a small amount, from 54.6 to 55.0A, to facilitate coincidence. This particular NCSL configuration is associated with a residual misfit, $\mathrm{F}$, calculated, to be about $+0.7 \%$, following:

$$
F=\frac{n a_{\mathrm{BaO}}-m a_{\mathrm{MgO}}}{\frac{1}{2}\left(n a_{\mathrm{BaO}}+m a_{\mathrm{MgO}}\right)}
$$


where $\mathrm{n}$ is the number of $\mathrm{BaO}$ unit cells (equal to 10) and $\mathrm{m}$, the number of $\mathrm{MgO}$ unit cells (equal to 13). Clearly, the energy required to accommodate a $0.7 \%$ misfit is much lower that that required to accommodate the full $27 \%$ misfit associated with the fully coherent system (i.e. $n=m=1$ ).

In addition to coincidences found by matching integer unit cells of the thin film with that of the substrate, coincidence can also be found by rotating one material with respect to the other. For the simplest case - where the thin film is the same material as the substrate - a twist grain-boundary will result [Duffy DM 1986]. For the $\mathrm{BaO} / \mathrm{MgO}$ system, a configuration associated with a low misfit and favourable interfacial interactions, involves a $45^{\circ}$ rotation of the $\mathrm{BaO}$ overlayer with respect to the $\mathrm{MgO}$ substrate and was observed experimentally by Cotter and coworkers [Cotter M 1988]; atomistic models for this structure have also been generated [Sayle DC 2002b].

\subsubsection{2 $\mathrm{CeO}_{2} / \mathrm{Al}_{2} \mathrm{O}_{3}$}

When a thin film is supported on a substrate, the supported thin film will respond structurally to the substrate to facilitate a low-energy configuration. This can include, for example, dislocation evolution and will influence the defect chemistry of the system. In this respect Sayle and coworkers explored how easy it was to extract an oxygen ion from the surface of a $\mathrm{CeO}_{2}$ thin film, when supported on an $\mathrm{Al}_{2} \mathrm{O}_{3}$ substrate, compared with oxygen extraction from the parent material [Sayle DC 1995]. The calculations suggested that supporting the $\mathrm{CeO}_{2}$ thin film on top of $\mathrm{Al}_{2} \mathrm{O}_{3}$ might promote the migration of oxygen from the interfacial region to the surface, a process of catalytic importance.

\subsubsection{3 $\mathrm{NiO} / \mathrm{ZrO}_{2}$}

Fisher and Matsubara extended the NCSL/atomistic simulation approach to generate heterophase interfaces between materials with rectangular surface symmetries and used the method to simulate eight heterophase interfaces between $\mathrm{NiO}$ and cubic $\mathrm{ZrO}_{2}$ [Fisher CAJ 2005]. They observed that many of the interfaces were found to comprise disordered, open structures with correspondingly high energies as a consequence of the strong repulsive forces between like-charged ions in close proximity. The exception was the $\mathrm{NiO}(111) / \mathrm{ZrO}_{2}(100)$, which was observed to exhibit good coherency across the interphase boundary, where the $\mathrm{NiO}$ and $\mathrm{ZrO}_{2}$ shared an oxygen plane. The rationale underlying this study was to demonstrate the ability of simulation-based methods for identifying interface configurations associated with strong cohesive forces holding the materials together for optimizing the properties of composites of technologically important materials.

A limitation with using a 'NCSL strategy' is that the simulator has to decide which particular NCSL to construct and then use atomistic simulation (i.e. energy minimization and or MD) to determine whether the configuration is of low-energy and therefore likely to be structurally realistic. An alternative is to use an evolutionary method to guide the generation of the interfacial configurations and structures. These methods, which includes simulated ion deposition section 9.4.1, do not require (or even allow!) the simulator to pre-specify a particular interfacial configuration.

\subsubsection{Amorphisation and Recrystallisation}

Here, the oxide thin film is placed on top of an oxide substrate and then amorphised. MD simulation, applied to this system (amorphous thin film on top of crystalline substrate) for long duration, results in the recrystallisation of the thin film [Sayle 1999]. In contrast to the recrystallisation process observed for 'isolated' nanoparticles in section 9.3, the underlying (crystalline) substrate is central in directing the structure of the overlaying (amorphous) thin film. In particular, the structure of the substrate facilitates the crystallization of the thin films by acting as a pseudo 'nucleating seed'. In addition, the simulator does not have to wait for a crystalline seed to 
spontaneously evolve from the amorphous sea of ions and therefore the simulation time, and hence computational cost, is reduced.

\subsubsection{1 $\mathrm{SrO} / \mathrm{MgO}(100)$}

Snapshots illustrating the A\&R process as applied to an $\mathrm{SrO}$ thin film, about $1 \mathrm{~nm}$ thick, supported on an $\mathrm{MgO}(001)$ substrate, are presented in fig. 9.10. Inspection of the final crystalline structure, fig. 9.10(d), using molecular graphics, revealed that the $\mathrm{SrO}$ thin film generated conformed to 8 lattice spacings for the overlying $\mathrm{SrO}$ thin film, lattice matched with 10 lattice spacings of the underlying $\mathrm{MgO}$ substrate (NCSL configuration). This supercell corresponds to a lattice misfit of about $-2 \%$, based upon the lattice parameters of the component materials: $\mathrm{a}_{\mathrm{SrO}}=2.57 \AA ; \mathrm{a}_{\mathrm{MgO}}=2.10 \AA$, which is much smaller that the 'bulk' misfit $(+20 \%)$. In addition, mixed screw-edge dislocations, shown in fig. 9.11, were observed to have evolved within the $\mathrm{SrO}$ thin film, which help both to accommodate the misfit associated between the thin film and underlying support and to remove deleterious interfacial interactions such as cations in close proximity [Sayle DC 2000].

\subsubsection{2 $\mathrm{MgO} / \mathrm{BaO}(100)$}

Figure 9.12 shows the structure of a thin film of $\mathrm{MgO}$ supported on a $\mathrm{BaO}(100)$ substrate. Here the recrystallisation resulted in the evolution of a nanopolycrystalline $\mathrm{MgO}$ film. The nanocrystallites range from about 200 to $2,000 \AA^{2}$ in size and are rotated, with respect to the underlying $\mathrm{BaO}$ surface normal by various angles. The structure of the grain-boundaries that are formed between neighbouring grains are consistent with grain-boundaries observed experimentally [Yan Y 1998, Pennycook SJ 1999]. An STEM image of an MgO grain-boundary is included in the figure for comparison and has been reproduced with permission [Pennycook SJ 1999]). Further details of this study can be found in [Sayle DC 2000b].

\subsubsection{3 $\mathrm{CeO}_{2} / \mathrm{YSZ}$}

In section 9.3.2 on $\mathrm{CeO}_{2}$ nanoparticles, it was suggested that by traversing down to the nanoscale, one is able to increase the proportion of reactive to unreactive $\mathrm{CeO}_{2}$ surfaces simply by virtue of the fact that if one goes small enough, then the number of oxygen ions accommodating reactive step/corner sites will be commensurate to oxygen ions comprising more stable positions and therefore unreactive surfaces. An alternative strategy is to fabricate thin films with nanoscale thicknesses. In particular, if a material, which is deposited onto a substrate, is sufficiently thin, then it is likely that the substrate will act as a template in directing the structure of the thin-film deposited thereon - including the particular surface it exposes.

For example, the (111) surface is energetically the most stable ceria surface. It is therefore exposed preferentially compared with less stable surfaces [Gritschneder S 2005]. However, the energy required to create oxygen vacancies on the (111) surface, which is directly linked to its catalytic activity [Sayle TXT 1992, 1994], is higher compared with the (110) surface. One therefore desires a mechanism for fabricating $\mathrm{CeO}_{2}(110)$ in preference to $\mathrm{CeO}_{2}(111)$. One way is to traverse to the nanoscale, as described previously, the other is to deposit a ultra-thin film of $\mathrm{CeO}_{2}$ on top of a substrate material. The rationale underlying the latter is that the substrate may act as a template [Sayle DC 2002a] in directing the structure of the overlying thin $\mathrm{CeO}_{2}$ film. A simulation study was therefore performed to explore this possibility.

A $\mathrm{CeO}_{2}$ film, about 2nm thick, was deposited on top of an YSZ(110) substrate (YSZ: yttrium stabilized zirconia). The $\mathrm{CeO}_{2}$ was first amorphised and then recrystallised. Full details of the simulation can be found in reference [Sayle DC 2002c]. It was anticipated that the YSZ(110) exposed at the surface would direct the $\mathrm{CeO}_{2}$ thin film to expose the (catalytically active) (110) surface also. 
Fig. 9.13 shows graphically the atom positions comprising the $\mathrm{CeO}_{2} / \mathrm{YSZ}(110)$ system. Inspection of these figures revealed that the YSZ(110) substrate did indeed influence the structure of the $\mathrm{CeO}_{2}$ thin film. However, surprisingly, while the $\mathrm{CeO}_{2}$ exposed the (110) surface as anticipated, it exposed also the (111) and dipolar (100). In particular, the $\mathrm{CeO}_{2}$ thin film overlayer was observed to be nanopolycrystalline with each of the nanocrystalline domains exposing a particular face at the surface - either (110), (100) or (111). It was proposed that the lattice misfit between the YSZ substrate and $\mathrm{CeO}_{2}$ thin film deposited thereon was in part responsible for the nanopolycrystalline structure of the $\mathrm{CeO}_{2}$ thin film.

\subsubsection{Melting and Seeded-Crystallisation}

Similar to A\&R, this evolutionary strategy involves crystallization from a melt. However, to reduce the computational time required for the system to evolve 'naturally' a crystalline seed, seeds were manually introduced into the liquid.

\subsubsection{FeO(nanocrystalline)/FeO}

A study by Phillpot and co-workers describe a simulation strategy for generating models for nanopolycrystalline $\mathrm{FeO}$ with grain-sizes of about 5nm using MD simulation [Phillpot SR 1999]. In particular the authors deposited an ultra thin, molten film of $\mathrm{FeO}$ on top of an $\mathrm{FeO}$ substrate. Within the molten film they introduced sixteen randomly oriented crystalline 'seeds'. The system was then cooled gradually under MD. During the crystallization, the (molten) Fe and O ions condensed onto the seeds propagating their structure and, because the seeds were misoriented, the crystallization resulted in the formation of a nanopolycrystalline film with general grain-boundaries as propagated by the orientation of the seeds. Inspection of the grain-boundaries revealed them to be structurally realistic - similar to those observed experimentally [Sutton AP 1995].

\subsubsection{Temperature Assisted Dynamics}

Another strategy for overcoming the debilitatingly short timescales accessible using MD simulations is Temperature Assisted Dynamics (TAD). This strategy is derived from a variety of methods labeled 'hyperdynamics', which were developed by Voter and co-workers [Voter AF 2002]. The strategy involves the use of simulations, performed at high temperatures, to gauge evolution of a system at a lower (realistic) temperature. For example, the energy required for an ion to move out of the surface of a material is so high that the probability of it happening at room temperature - and within timescales accessible to MD - is low. Conversely, if we increase the temperature, the probability of it occurring within the same timescale increases. Accordingly, the simulation is run at sufficiently high a temperature to ensure the process occurs with the timescale accessible. Once the transition is detected (i.e. the ion moves out of the surface), the attributes of this process are recorded and characterized. This process, together all other related important processes, are then transferred back to a simulation performed at a more realistic (low) temperature. For a more comprehensive treatment of the method see [Voter AF 2002].

\subsubsection{BaO/SrO}

Harris and co-workers used TAD to explore the surface diffusion and heteroepitaxial growth of $\mathrm{BaO}$ on an $\mathrm{SrO}$ substrate. Crucially, it was found that the surface diffusion proceeds most favourably by an exchange mechanism involving the surface layer. For example, as a barium ion diffuses across the SrO surface, it was found that an energetically favourable pathway, in addition to a simple hopping across the surface, is for the barium ion to swap places with a strontium ion located within the surface atomic layer of the underlying SrO substrate [Harris DJ 2004, 2005]. This has severe implications for growing sharp interfaces between different oxide materials using, for example, molecular beam epitaxy. Mixing can also lead to surface contamination and subsequent 
changes in surface properties. It is likely that this phenomenon will occur also at the surface of nanomaterials - nanoparticles and thin films, which will present problems to applications utilizing nanodevices.

\subsection{Supported Nanoparticles}

Thus far we have seen that changing one or more of the dimensions of a system to the nanoscale can influence, sometimes profoundly, its microstructure and hence properties. Clearly it is important to be able to exercise control over the structure to fine tune or indeed optimize desirable properties [Sata N 2000]. To this end one can envisage supporting oxide nanoparticles on an oxide substrate: Indeed, it is well-known that small metal particles, supported on an oxide substrate, can be catalytically more active compared with the parent materials [Diebold U 2003].

\subsubsection{MO/BaO (M= Sr, $\mathrm{Ca}, \mathrm{Mg})$}

Models for $\mathrm{SrO}, \mathrm{CaO}$ and $\mathrm{MgO}$ nanoparticles were generated by placing a rectangular 25,000-atom block of each oxide onto the surface of a $\mathrm{BaO}(100)$ substrate. The oxide nanoparticles were then amorphised and recrystallised. Further details can be found in ref. [Sayle DC 2002d]. During the recrystallisation, the interactions between the nanoparticle and the underlying $\mathrm{BaO}$ substrate were found to have a profound influence on the structure of the nanoparticle. Indeed, these interactions facilitate a wealth of microstructural features that evolve during the recrystallisation.

For the $\mathrm{SrO} / \mathrm{BaO}(100)$ system ("low" $-7 \%$ misfit - equation 9.10), the $\mathrm{SrO}$ nanoparticle was found to lie coherent with the underlying $\mathrm{BaO}$ substrate. For the $\mathrm{CaO} / \mathrm{BaO}(100)$ system ("medium" $-15 \%$ misfit) only small regions of coherence was observed between the $\mathrm{CaO}$ and $\mathrm{BaO}$ with dislocations evolving within $\mathrm{CaO}$ regions that were misaligned with respect to the underlying $\mathrm{BaO}$. For the $\mathrm{MgO} / \mathrm{BaO}$ system ("high" $-31 \%$ misfit), the misfit is so high that no regions of coherence between the $\mathrm{MgO}$ nanoparticle and $\mathrm{BaO}$ substrate could be identified. This study also proposed the existence of a critical area for dislocation; the concept of a critical thickness is well-known [Sayle DC 2002d]. If confirmed experimentally, this prediction may have important implications with respect to the field of microelectronic circuits.

In fig. 9.14, the structure of the $\mathrm{MgO} / \mathrm{BaO}(100)$ system is shown. Close inspection of the nanoparticle reveals a variety of microstructural features, including:

(i) Morphology The nanoparticle is not a rectangular block, rather it comprises four crystallites interconnecting at various angles. The central region, coloured red, exposes the $\mathrm{MgO}(100)$ at the surface and at the interface, i.e. $\mathrm{MgO}(100) / \mathrm{BaO}(100)$. The missoriented crystallites, coloured yellow, blue and grey, emanating from this central region, appear to exhibit triangular pyramidal morphologies. The nanoparticle exhibits a high density of surface steps.

(ii) Grain-boundaries The four interconnecting crystallites, are misaligned, which gives rise to various grain boundary structures. Inspection of these structures in fig. 9.14(a) reveals that they are complex (general) and therefore difficult to assign to a particular coincidence site lattice (CSL) description. Moreover, bending of the lattice planes compounds this difficulty.

(iii) Epitaxial Configurations The crystallites expose the $\mathrm{MgO}(100)$ and $\mathrm{MgO}(111)$ surfaces at the interface (we note that rocksalt $\{111\}$ surfaces are dipolar). No epitaxial configuration could be identified. This is attributed to the high misfit associated with this system.

(iv) Point defects The interfacial region, fig. 9.14(b), is highly defective and includes voids, vacancies and cation intermixing across the $\mathrm{MgO} / \mathrm{BaO}$ boundary. 
(v) Decorations Inspection of fig. 9.14(a) reveals that the surface of the $\mathrm{MgO}$ nanoparticle is decorated with many $\mathrm{Ba}$ ions that have migrated from the $\mathrm{BaO}$ substrate.

(vi) Dislocations Dislocations were observed to have evolved within the $\mathrm{MgO}$ nanoparticle, the core structure of a mixed screw-edge dislocation is shown in fig. 9.14(c)

This study provides an illustration that supporting a oxide nanoparticle, on an oxide substrate, can influence profoundly the structure and microstructure of the nanoparticle and is likely to modify its properties.

\subsection{Encapsulated Nanoparticles}

An important area within nanoscience is the embedding of nanoparticles within a host lattice. For example various materials have been encapsulated within microporous materials such as zeolites [Viswanadham N 2001]. More recently, materials have been encapsulated within single and multiwall carbon nanotubes [Monthioux M 2002], leading to the fabrication of one-dimensional materials. The intense scientific interest in these systems is hardly surprising since with appropriate choice of encapsulating lattice, one can potentially exact control over the structure and properties of the encapsulated material. This has inspired many researchers to explore materials that might act as the host lattice and materials that might prove suitable to be encapsulated. By mixing and matching, an almost limitless number of possible systems can be envisaged. One can also envisage encapsulation of a nanoparticle within a fully dense host lattice. Indeed, Jeng and Shen have explored the structure of $\mathrm{NiO}$ nanoparticles encapsulated within $\mathrm{CaO}$ by sintering and annealing $\mathrm{NiO}$ and $\mathrm{CaO}$ powders [Jeng ML 2000]. Atomistic simulation can also be used to predict the structure and morphology of encapsulated nanoparticles. One such study by Sayle and Parker, explored the influence of introducing an oxide nanoparticle into the near surface region of an oxide host [Sayle DC 2003b]. In particular, models for oxide encapsulated oxide nanoparticles were generated by constructing a block of a rocksalt-structured oxide. The surface region of this block, comprising about 25,000 atoms, was then amorphised Once amorphous, a spherical nanoparticle about 700 atoms in size, was introduced. The whole system was then recrystallised and the system analysed structurally. The procedure is illustrated, for $\mathrm{CaO}$ encapsulation within an $\mathrm{MgO}$ host, in fig. 9.15 .

The introduction of the amorphous $\mathrm{CaO}$ nanoparticle into the amorphous $\mathrm{MgO}$ substrate is shown in fig. 9.15(a). Fig. 9.15(b) shows the system after recrystallisation. In this figure, the $\mathrm{CaO}$ nanoparticle has been extracted out of the host lattice to show more clearly the morphology of the nanoparticle and the void, within the MgO host, it occupies. Fig. 9.15(c) shows the system with a segment of the simulation cell cut-away. This enables one to see more clearly the nanoparticle within the host lattice. In fig. 9.15(d), a slice cut through the system is shown to reveal that the $\mathrm{CaO}$ nanoparticle rotates slightly with respect to the $\mathrm{MgO}$ host. The driving force for this to occur was attributed to reducing the lattice misfit associated with the configuration, whilst maximising favourable cation-anion interactions across the (curved) interfacial region. Careful analysis, using graphical techniques, reveals that the $\mathrm{CaO}$ nanoparticle exposes $\{100\},\{110\}$ and $\{111\}$ facets to the $\mathrm{MgO}$ host at the interfacial regions. In addition, this procedure was performed for $(\mathrm{BaO}$ and $\mathrm{CaO}$ ) nanoparticles encapsulated in $\mathrm{MgO}$, and $(\mathrm{SrO}$ and $\mathrm{MgO}$ ) nanoparticles encapsulated in $\mathrm{BaO}$. The structure of $\mathrm{MgO}, \mathrm{BaO}, \mathrm{CaO}$ and $\mathrm{SrO}$ nanoparticles, which have been extracted out of their respective host lattices to reveal more clearly the morphology they accommodate, are shown in fig. 9.16(a-d) respectively. 
The study raised several issues pertaining to the encapsulation of nanoparticles. In particular, the nanoparticles were identified to be highly strained. For example, the $\mathrm{SrO}$ nanoparticle, encapsulated within $\mathrm{BaO}$, revealed that the $\mathrm{SrO}$ maintained full alignment of counter ions across the interfacial regions. The lattice misfit associated with the $\mathrm{SrO} / \mathrm{BaO}$ system is about $-7 \%$ and therefore the $\mathrm{SrO}$ is tensioned to maintain this aligned configuration. It was proposed that the energetically favourable cation-anion interactions across the interfacial region outweighed the energetically deleterious effect of tensioning the lattice. Clearly, if the size of the nanoparticle were increased then the energy required to tension the lattice, which operates effectively in 3-dimensions, would increase faster than the increase in the favourable interfacial interactions, which is a surface phenomenon and inherently two-dimensional. This suggests that there is a 'critical' size associated with encapsulated nanoparticles, above which misfit dislocations evolve. Indeed, dislocations/commensurate structures were observed for the other three systems, which are all associated with a higher lattice misfit. The critical size was predicted to depend upon a combination of both the nanoparticle and the host within which it is encapsulated.

In summary, the encapsulated nanoparticles exhibited a range of morphologies, expose a variety of facets at the nanoparticle/host lattice interface, and are observed to rotate within the cavity in which they occupy. The structure and nature of the nanoparticles reflect the lattice misfit between the nanoparticle and the host lattice. Further details can be found in [Sayle DC 2003b]

\subsection{Conclusions}

In this chapter we have explored primarily how models of oxide nanomaterials, with full atomistic detail, can be generated using computer simulation. These include isolated nanoparticles, ultra-thin films supported on a substrate, supported nanoparticles and encapsulated nanoparticles. Once a model is available, a wealth of electronic, chemical and mechanical properties can then be calculated and the results used predictively to aid experiment: Properties amenable to calculation include, for example, diffusion coefficients, ionic conductivity [Sayle DC 2005], catalytic activity (included in this chapter), elastic constants, bulk and shear moduli, Youngs moduli, piezoelectric constants, phonons, dielectric constants, surface energies [Gay DH 1995]. A well-established and popular computer code for calculating a variety of properties using such atomistic models is the General Utility Lattice Program (GULP), which is freely available from the Author [Gale JD 2003].

The atomistic models derived are also useful as starting structures for Quantum Mechanical (QM) simulations. This is because it is less expensive computationally to generate models using atomistic simulation compared with QM

It is tempting to think, especially when viewing the animations of $\mathrm{MnO}_{2}$ crystallising (fig. 9.3), and the resulting nanoparticle structures, that one is observing 'real' crystallisation at the atomistic level. However, one must exercise caution in that the simulation is bound by (artificial) simulation constraints implicit in the methodology - not least by the potential models describing the interactions between the ions. Conversely, it is very surprising that such highly-complex microstructural features, observed experimentally, such as the dislocations, grain-boundaries, morphologies, surface structures, isolated and associated point defects, have simply evolved in a purely artificial way within the simulations described above. Accordingly, we suggest that the simulated Amorphisation and Recrystallisation (A\&R) we have discussed in this chapter, must, at least in part, reflect crystallisation that occurs in nature, and that the models generated using this approach are realistic. 
If used with a degree of caution, models generated using atomistic simulation can help experiment unravel the rich structural complexity of nanomaterials and aid the fabrication of nanomaterials with improved, tunable or indeed new properties.

\section{References}

Chabre Y, Pannetier J (1995). Structural and Electrochemical Properties of the Proton GammaMNO2 system. Progress in Solid State Chemistry 23: 1-130

Chambers SA (2000). Epitaxial growth and properties of thin film oxides. Surface Science Reports 39: 105-180.

Chen H-I, Chang H-Y (2005). Synthesis and characterization of nanocrystalline cerium oxide powders by two-stage non-isothermal precipitation. Solid State Communications 133: 593-598.

Cordatos H, Ford D, Gorte RJ (1996). Simulated annealing study of the structure and reducibility in ceria clusters. Journal of Physical Chemistry 100: 18128-18132

Cotter M, Campbell S, Egdell RG. (1988). Growth of ordered BaO overlayers on MgO (001). Surface Science 197: 208-224.

Diebold U (2003). The surface science of titanium dioxide. Surface Science Reports 48: 53-229.

Dong L, Schnitker J, Smith RW, Srolovitz DJ (1998). Stress relaxation and misfit dislocation nucleation in the growth of misfitting films: A molecular dynamics simulation study. Journal of applied physics 83: 217-227.

Duffy DM, Harding JH (2004). Growth of polar crystal surfaces on ionized organic substrates. Langmuir 20 : 7637-7642.

Duffy DM (1986). Grain boundaries in ionic crystals. Journal of Physics C - Solid state Physics 19: 4393-4412.

Fisher CAJ, Matsubara H (2005). Molecular dynamics simulations of interfaces between $\mathrm{NiO}$ and cubic ZrO2. Philosophical Magazine 85: 1067-1088.

Gale JD, Rohl AL (2003). The General Utility Lattice Program (GULP). Molecular Simulation 29: 291-341.

Gale JD, Rohl AL (1995). MARVIN - A new computer code for studying Surfaces and Interfaces and its application to calculating the crystal morphologies of Corundum and Zirconia. Journal of the Chemical Society - Faraday Transactions 91: 925-936.

Gritschneder S, Namai Y, Iwasawa Y, Reichling M (2005). Structural features of CeO2(111) revealed by dynamic SFM. Nanotechnology 16: S41-S48 Sp.

Hamad S, Cristol S, Catlow CRA (2005). Simulation of the embryonic stage of ZnS formation from aqueous solution. Journal of the American Chemical 127 (8): 2580-2590. 
Harding JH (1999). The simulation of general polar boundaries. Surface Science 422: 87-94.

Harris DJ, Lavrentiev MY, Harding JH, Allan NL, Purton JA (2004). Novel exchange mechanisms in the surface diffusion of oxides. Journal of Physics-Condensed Matter 16: L187-L192.

Harris DJ, Farrow TS, Harding JH, Lavrentiev MY, Allan NL, Smith W, Purton JA (2005). Surface diffusion and surface growth in nanofilms of mixed rocksalt oxides. Physical Chemistry Chemical Physics 7: 1839-1844.

Hill MR, Freeman CM, Rossouw MH (2004). Understanding gamma-MnO2 by molecular modeling. Journal of Solid State Chemistry 177: 165-175.

Jeng ML, Shen PY (2000). Thermally activated rotation of Ni1-xO particles within $\mathrm{CaO}$ grains. Materials Science and Engineering - A structural Materials properties Microstructure and Processing 287: 1-9.

Johnston RL (2003). Evolving better nanoparticles: Genetic algorithms for optimising cluster geometries. Dalton Transactions 22: 4193-4207.

Leach AR (2001). Molecular Modelling, Principles and applications. Second edition. Copyright Pearson Education Limited. ISBN 0-582-38210-6

Lewis GV, Catlow CRA (1985). Potential Models for Ionic Oxides. Journal of Physics C-- Solid State Physics 18: 1149-1161.

Lu YF, Przybylski M, Trushin O, Wang WH, Barthel J, Granato E, Ying SC, Ala-Nissila T (2005). Strain relief in Cu-Pd heteroepitaxy. Physical Review Letters 94 : Article. no. 146105.

Mohn CE, Lavrentiev MY, Allan NL, Bakken E, Stolen S (2005). Size mismatch effects in oxide solid solutions using Monte Carlo and configurational averaging. Physical Chemistry Chemical Physics 7: 1127-1135.

Di Monte R, Kaspar J (2005). Nanostructured CeO2-ZrO2 mixed oxides. Journal of Materials Chemistry 15: 633-648

Monthioux M (2002). Filling single-wall carbon nanotubes. CARBON 40: 1809-1823.

Norenberg H, Harding JH (2001). The surface structure of CeO2(001) single crystals studied by elevated temperature STM. Surface Science 477: 17-24.

Pennycook SJ, Dickey EC, Nellist PD, Chisholm MF, Yan Y, Pantelides ST (1999). A combined experimental and theoretical approach to atomic structure and segregation at ceramic interfaces. Journal of the European ceramic society. 19: 2211-2216.

Phillpot SR, Keblinski P, Wolf D, Cleri F (1999). Synthesis and characterization of a polycrystalline ionic thin film by large-scale molecular-dynamics simulation. Interface Science 7: 15-31. 
Piana S, Gale JD (2005). Understanding the barriers to crystal growth: Dynamical simulation of the dissolution and growth of urea from aqueous solution. Journal of the American Chemical Society. 127: 1975-1982.

Rohl AL (2003). Computer prediction of crystal morphology. Current opinion in Solid State \$ Material Science 7: 21-26.

Sata N, Eberman K, Eberl K, Maier J (2000). Mesoscopic fast ion conduction in nanometre-scale planar heterostructures. Nature 408: 946-949.

Sayle DC, Sayle TXT, Parker SC, Harding JH, Catlow CRA (1995). The stability of defects in the ceramic interfaces, $\mathrm{MgO} / \mathrm{MgO}$ and $\mathrm{CeO} 2 / \mathrm{Al} 2 \mathrm{O} 3$. Surface Science 334: 170-178.

Sayle DC, Maicaneanu SA, Slater B, Catlow CRA (1999a). Exercising control over the influence of the lattice misfit on the structure of oxide-oxide thin film interfaces. Journal of Materials Chemistry 9: 2779-2787.

Sayle DC (1999b). The predicted 3-D atomistic structure of an interfacial screw-edge dislocation. Journal of Materials Chemistry 9: 2961-2964 1999b

Sayle DC, Watson GW (2000a). Dislocations, lattice slip, defects and rotated domains: The effect of a lattice misfit on supported thin-film metal oxides. Physical Chemistry Chemical Physics. 2: 5491-5499

Sayle DC, Watson GW (2000b). Simulated amorphisation and recrystallisation: nanocrystallites within meso-scale supported oxides. Journal of Materials Chemistry 10: 2241-2243

Sayle DC, Watson GW (2002a). Inducing polycrystallinity within supported oxide thin films using template buffer layers. Journal of Physical Chemistry B 106: 3778-3787.

Sayle DC, Catlow CRA, Dulamita N, Healy MJF, Maicaneanu SA, Slater B and Watson GW (2002b). Modelling Oxide Thin-Films. Molecular Simulation, 28 (6-7), 683-725.

Sayle DC, Maicaneanu SA, Watson GW (2002c). Atomistic models for $\mathrm{CeO}_{2}(111)$, (110), and (100) nanoparticles, supported on yttrium-stabilized zirconia. Journal of the American Chemical Society 124: 11429-11439.

Sayle DC, Doig JA, Maicaneanu SA, Watson GW (2002d). Atomistic structure of oxide nanoparticles supported on an oxide substrate. Physical Review B 65: Article. no. 245414.

Sayle DC, Johnston RL (2003a). Evolutionary techniques in atomistic simulation: thin films and nanoparticles. Current Opinion in Solid State \& Materials Science 7: 3-12.

Sayle DC, Parker SC (2003b). Encapsulated oxide nanoparticles: The influence of the microstructure on associated impurities within a material. Journal of the American Chemical Society 125: 8581-8588. 
Sayle DC, Doig JA, Parker SC, Watson GW, Sayle TXT (2005). Computer aided design of nanostructured materials with tailored ionic conductivities. Physical Chemistry Chemical Physics 7: 1618.

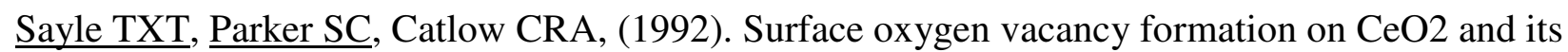
role in the oxidation of Carbon Monoxide. The chemical Society - Chemical Communications 14: 977-978.

Sayle TXT, Catlow CRA, Sayle DC, Parker SC, Harding JH, (1993). Computer - Simulation of thin-film heteroepitaxial ceramic interfaces using a near-coincidence-site lattice theory.

Philosophical magazine A - Physics of condensed matter structure defects and mechanical properties 68: 565-573.

Sayle TXT, Parker SC, Catlow CRA (1994). The role of oxygen vacancies on Ceria surfaces in the oxidation of carbon monoxide. Surface Science 316: 329-336

Sayle TXT, Parker SC, Sayle DC (2004). Shape of CeO2 nanoparticles using simulated amorphisation and recrystallisation. Journal of the chemical Society - Chemical Communications 21: 2438-2439.

Sayle TXT, Parker SC, Sayle DC (2005a). Oxidising CO to $\mathrm{CO}_{2}$ using ceria nanoparticles. Physical Chemistry Chemical Physics 7: 2936-2941.

Sayle TXT, Catlow CRA, Maphanga RR, Ngoepe PE, Sayle DC (2005b).

Generating $\mathrm{MnO}_{2}$ Nanoparticles Using Simulated Amorphization and Recrystallisation

Journal of the American Chemical Society 127(37): 12828-12837; Dor: 10.1021/ja0434073

Schnitker J, Srolovitz DJ (1998). Misfit effects in adhesion calculations. Modelling and Simulation in Materials Science and Engineering 6: 153-164.

Schissel DP (2005). Grid computing and collaboration technology in support of fusion energy sciences. Physics of Plasmas 12 (5): Article no. 058104.

Smith W, Yong CW, Rodger PM (2002). DL_POLY: Application to molecular simulation. Molecular Simulation 28: 385-471.

Smith W, Forester TR (1996). DL_POLY is a package of Molecular Simulation routines written by Smith and Forester Copyright by the council for the Central Laboratory of the Research Councils, Daresbury Laboratory, Daresbury, Warrington, UK.

http://www.dl.ac.uk/TCSC/Software/DLPOLY.

Stanek CR, Bradford MR, Grimes RW (2004). Segregation of Ba2+, Sr2+, Ce4+ and Zr4+ to UO2 surfaces. Journal of Physics-Condensed Matter 16: S2699-S2714.

Sutton AP, Baluffi RW (1995). Interface in Crystalline Materials, Monographs on the Physics and Chemistry of Materials, vol. 51, Oxford University Press Inc., New York.

Stoneham AM (1985). Interatomic potentials for condensed matter-Physics A, B \&C 131: 69-73. 
Titiloye JO, Parker SC, Osguthorpe DJ, Mann S (1991). Predicting the influence of growth additives on morphology of ionic crystals. Journal of the chemical Society - Chemical Communications 20: 1494-1496.

Viswanadham N, Shido T, Iwasawa Y (2001). Performances of rhenium oxide-encapsulated ZSM-5 catalysts in propene selective oxidation/ammoxidation. Applied Catalysis A General 219: 223-233.

Voter AF, Montalenti F, Germann TC (2002). Extending the time scale in atomistic simulation of materials. Annual Review of Materials Research 32: 321-346.

Vyas S, Grimes RW, Gay DH, Rohl AL (1998). Structure, stability and morphology of stoichiometric ceria crystallites. Journal of the chemical Society -Faraday Transactions 94: 427434.

Walker AM, Slater B, Gale JD, Wright K (2004). Predicting the structure of screw dislocations in nanoporous materials. Nature materials 3: 715-720.

Wang ZL, Feng XD (2003). Polyhedral shapes of CeO2 nanoparticles. Journal of Physical Chemistry B-107: 13563-13566.

Yan Y, Chisholm MF, Duscher G, Maiti A, Pennycook SJ, Pantelides ST (1998). Impurity-induced structural transformation of a MgO grain boundary. Physical Review Letters 17: 3675-3678.

Yu HB, Kim JH, Lee HI, Scibioh MA, Lee J, Han J, Yoon SP, Ha HY (2005). Development of nanophase $\mathrm{CeO} 2-\mathrm{Pt} / \mathrm{C}$ cathode catalyst for direct methanol fuel cell. Journal of power sources 140 : $59-65$.

Zhang F, Jin Q, Chan SW (2004). Ceria nanoparticles: Size, size distribution, and shape. Journal of Applied Physics 95: 4319-4326. 